\title{
Telerounding \& telementoring for urological procedures
}

\author{
Volkan Sen, Ozgu Aydogdu, Tarik Yonguc, Ibrahim Halil Bozkurt, Deniz Bolat \\ Izmir Bozyaka Training and Research Hospital, Department of Urology Izmir, Turkey.
}

\begin{abstract}
Summary Telemedicine is the use of medical information exchanged from one site to another via electronic communications to improve a patient's clinical health status. Telemedicine includes a growing variety of applications and services using two-way video, email, smart phones, wireless tools and other forms of telecommunications technology. Starting out over forty years ago with demonstrations of hospitals extending care to patients in remote areas, the use of telemedicine has spread rapidly and is now becoming integrated into the ongoing operations of hospitals, specialty departments, home health agencies, private physician offices as well as consumer's homes and workplaces. There's also a current trend in the use of telemedicine in urology. In the present paper we aimed to review the recent literature about telemedicine and the use of telerounding and telementoring in urological procedures.
\end{abstract}

KEY WORDS: Telemedicine; Telementoring; Telerounding.

Submitted 23 February 2016; Accepted 3 March 2016

\section{INTRODUCTION}

Telemedicine had come to the fore with the report by Aronson SH at 1977 about telephone using (1).

Subsequent studies about telemedicine were performed on e-mail $(2,3)$. Johansen MA et al. investigated whether the parents of burns patients could capture suitable clinical images with a digital camera and add the necessary text information to enable the pediatric burns team to provide follow-up care via email and they suggested that this technique could be used as a low-cost telemedicine service in burns follow-up (2). Telerounding is described as remote patient rounding by using computers, laptops, cameras and tablets. It was firstly reported in 2004 by Ellison et al. using a computer with a camera for telerounding installed on a remotely controlled custom service robot platform with 85 patients (3). They emphasized that the patients in the telerounding arm demonstrated statistically substantial improvements in ratings of examination thoroughness, quality of discussions about medical information, postoperative care coordination, and attending physician availability. Further trials supported the findings of these studies and telemedicine has been also widely used in urology clinics (4-6). The recent paper focused on the use of telemedicine in urology practice.

\section{TELEROUNDING IN UROLOGY}

In 2007 a large multi-institutional randomized study by Ellison et al., included 270 patients; 134 in the robotic telerounding arm and 136 patients in bedside round arm, demonstrated high rates of patient satisfaction in telerounding arm (4). Patients undergoing the following laparoscopic procedures were offered participation in this study: nephrectomy, partial nephrectomy, nephroureterectomy, retroperitoneal lymph node dissection, partial ureterectomy, and radical prostatectomy. Forty-five percent of the patients assessed that their care was better because of telerounding, $67 \%$ said that it should be a regular part of patient care in the hospital, $86 \%$ could easily communicate with their doctor using the telerounding system, $76 \%$, if hospitalized again, would feel comfortable with telerounding, and $67 \%$ claimed that if their doctor was out of town they would rather teleround with their doctor than be seen by another doctor. There were no significant differences in hospitalization time and complication rates between the two arms.

Kau et al. were evaluated the telerounding system by laptop computers with built-in webcam and video conferencing software in 2008 (5). Ten physician, 14 nurses and 10 patients were included in the study; $90 \%$ of patients agreed that they could easily communicate with their physician using video-rounding system (VRS). All patients strongly agreed that VRS should be a regular part of patient care and that they would be comfortable using VRS if their physician was unable to be in direct contact with them. All physicians and nurses agreed that VRS was easy to use, enhanced patient care, would be a comfortable alternative if direct physician contact was not possible, and that it should be a regular part of institutional care.

With the technological developments, the tablets had begun to be used for telerounding. Kaczmarek et al. reported the first study with tablet telerounding in 2012 (6). Totally 32 postoperative patients ( 25 robotic partial nephrectomy, 2 radical nephrectomy, 3 robotic-assisted radical prostatectomy, 1 adrenalectomy and 1 nephroureterectomy) were evaluated in this study. Patients expressed a high level of satisfaction with $91 \%$ of patients stating that their care was better using telerounding and $97 \%$ of patients stating that telerounding should be a regular part of patient care in the hospital. Additionally, 94\% of patients stated that they could easily communicate with their doctor over the telerounding system, $84 \%$ of patients agreed that they would feel comfortable with telerounding daily if they were hospitalized again and $81 \%$ of patients would prefer telerounding communication with their doctor than be directly seen by another doctor. The results of the telerounding studies were summarized in Table 1. By the technological developments, telerounding systems have become more cost-effective and more available and afford- 
Table 1.

Summary of studies for telerounding systems.

\begin{tabular}{|c|c|c|c|c|}
\hline & Ellison et al, 2004 & Ellison et al, 2007 & Kau et al, 2008 & Kaczmarek et al, 2012 \\
\hline Number of patients & 85 & 270 & 10 & 32 \\
\hline Rate of patients who assessed that their care was better because of telerounding & $42.4 \% *$ & $45 \%$ & $60 \%$ & $91 \%$ \\
\hline Rate of patients who said that it should be a regular part of patient care in the hospital & $76.9 \%$ & $67 \%$ & $100 \%$ & $97 \%$ \\
\hline Rate of patients who could easily communicate with their doctor using the telerounding system & $80.8 \%$ & $86 \%$ & $90 \%$ & $94 \%$ \\
\hline Rate of patients who, if hospitalized again, would feel comfortable with telerounding & $66,6 \%$ & $76 \%$ & - & $84 \%$ \\
\hline
\end{tabular}

able. In recent years especially the tablets has an advantage of lower purchase cost, widespread availability, uncomplicated setup. Increased device portability inherent to its light weight and small size allows the device to be easily carried between floors and clinical locations.

The increased patients' satisfaction in Kaczmarek's study could be explained by these reasons. These studies showed us the telerounding system has potential benefit for the physician as well as the patient. Doctors can gain convenience and flexibility with the potential for overseeing more patients. The average time of about 5 minutes is enough to facilitate communication and patient satisfaction. But we wish to emphasize that any telerounding systems could not replace the bedside rounding, they should be only an additional visits for bedsides rounds.

\section{TELEMENTORING IN UROLOGY}

Advances in technology have opened new avenues for long-distance communication through telemedicine. With this technology, an expert surgeon can observe and actively supervise a procedure performed by a trainee surgeon at another institution. Telementoring is an application of telemedicine that involves the remote guidance of procedures when the operator has limited experience with the technique. The first instances of transcontinental urologic telesurgery performed using the da Vinci system was reported by Sterbis et al. in porcine model at 2007 (7). Nephrectomy was successfully performed in 4 animals without any complication. Robot-assisted radical prostatectomy (RARP) has a long learning curve. In recent studies this curve was reported in experienced open surgeons as 8 to 20 operations (8-10). This curve is higher in trainee surgeons therefore mentoring of trainee surgeons has become more important to ensure that they attain sufficient skill without compromising the safety of their initial patients. Mentoring is usually done face-to-face, which presents practical difficulties when the mentor and trainee belong to different institutions. A telementoring system for robot-assisted surgery was firstly tested by Hinata et al. in 30 patients which avoids expensive and time-consuming travel by mentors (11). They found that the perioperative outcome of RARP performed with telementoring was not inferior to direct mentoring.

\section{Conclusion}

These studies showed us that the telerounding system has potential benefit for the physician as well as the patient. Doctors can gain convenience and flexibility with the potential for overseeing more patients. However we wish to emphasize that any telerounding system could not replace the bedside rounding, but they should be only additional visits to bedsides rounds. The telementoring system for urological procedures seems to be reliable and feasible, however further comprehensive studies including larger patient cohorts are needed.

\section{REFERENCES}

1. Aronson SH. The Lancet on the telephone 1876-1975. Med Hist. 1977; 21:69-87.

2. Johansen MA, Wootton R, Kimble R, et al. A feasibility study of email communication between the patient's family and the specialist burns team. J Telemed Telecare 2004; 10 Suppl 1:53-56.

3. Ellison LM, Pinto PA, Kim F, et al. Telerounding and patient satisfaction after surgery. J Am Coll Surg. 2004; 199:523-530.

4. Ellison LM, Nguyen M, Fabrizio MD, et al. Postoperative robotic telerounding: a multicenter randomized assessment of patient outcomes and satisfaction. Arch Surg. 2007; 142:1177-1181.

5. Kau EL, Baranda DT, Hain P, et al. Video rounding system: a pilot study in patient care. J Endourol. 2008; 22:1179-82.

6. Kaczmarek BF, Trinh QD, Menon M, et al. Tablet telerounding. Urology 2012; 80:1383-8.

7. Sterbis JR, Hanly EJ, Herman BC, et al. Transcontinental telesurgical nephrectomy using the da Vinci robot in a porcine model. Urology 2008; 71:971-3.

8. Ahlering TE, Skarecky A, Lee D, et al. Successful transfer of open surgical skills to a laparoscopic environment using a robotic interface: initial experience with laparoscopic radical prostatectomy. J Urol. 2003; 170:1738-41.

9. Patel VR, Tully AS, Holmes R, et al. Robotic radical prostatectomy in the community setting - the learning curve and beyond: initial 200 cases. J Urol. 2005; 174:269-72.

10. Menon M, Shrivastava A, Kaul S, et al. Vattikuti Institute prostatectomy: contemporary technique and analysis of results. Eur Urol. 2007; 51:648-57.

11. Hinata N, Miyake H, Kurahashi T, et al. Novel telementoring system for robot-assisted radical prostatectomy: impact on the learning curve. Urology 2014; 83:1088-92.

\section{Correspondence}

Volkan Sen, MD (Corresponding Author) - sen_volkan@yahoo.com Ozgu Aydogdu, MD - ozgucan@yahoo.com Tarik Yonguc, MD - tyonguc@gmail.com Ibrahim Halil Bozkurt, MD - ihalilbozkurt@yahoo.com Deniz Bolat, MD - drbolat@hotmail.com

Department of Urology, Izmir Bozyaka Training and Research Hospital, Izmir, Turkey 